ARTICLE

Received 28 Nov 2015 | Accepted 29 Mar 2016 | Published 9 May 2016

DOl: $10.1038 /$ ncomms11441

OPEN

\title{
Visualizing non-equilibrium lithiation of spinel oxide via in situ transmission electron microscopy
}

Kai He ${ }^{1, \star}$, Sen Zhang ${ }^{2, \star}$, Jing $\mathrm{Li}^{1}$, Xiqian $\mathrm{Yu}^{1}$, Qingping Meng${ }^{1}$, Yizhou Zhu ${ }^{3}$, Enyuan Hu${ }^{1}$, Ke Sun ${ }^{1}$, Hongseok Yun ${ }^{2}$, Xiao-Qing Yang ${ }^{1}$, Yimei Zhu' ${ }^{1}$, Hong Gan ${ }^{1}$, Yifei Mo ${ }^{3}$, Eric A. Stach ${ }^{1}$, Christopher B. Murray ${ }^{2} \&$ Dong Su ${ }^{1}$

Spinel transition metal oxides are important electrode materials for lithium-ion batteries, whose lithiation undergoes a two-step reaction, whereby intercalation and conversion occur in a sequential manner. These two reactions are known to have distinct reaction dynamics, but it is unclear how their kinetics affects the overall electrochemical response. Here we explore the lithiation of nanosized magnetite by employing a strain-sensitive, bright-field scanning transmission electron microscopy approach. This method allows direct, real-time, high-resolution visualization of how lithiation proceeds along specific reaction pathways. We find that the initial intercalation process follows a two-phase reaction sequence, whereas further lithiation leads to the coexistence of three distinct phases within single nanoparticles, which has not been previously reported to the best of our knowledge. We use phase-field theory to model and describe these non-equilibrium reaction pathways, and to directly correlate the observed phase evolution with the battery's discharge performance.

\footnotetext{
${ }^{1}$ Brookhaven National Laboratory, Upton, New York 11973, USA. ${ }^{2}$ Department of Chemistry, University of Pennsylvania, Philadelphia, Pennsylvania 19104, USA. ${ }^{3}$ Department of Materials Science and Engineering, University of Maryland, College Park, Maryland 20742, USA. * These authors contributed equally to this work. Correspondence and requests for materials should be addressed to C.B.M. (email: cbmurray@sas.upenn.edu) or to D.S. (email: dsu@bnl.gov).
} 
T he spinel transition metal oxide family-which includes $\mathrm{LiMn}_{2} \mathrm{O}_{4}, \quad \mathrm{Li}_{4} \mathrm{Ti}_{5} \mathrm{O}_{12}, \quad \mathrm{Fe}_{3} \mathrm{O}_{4}, \quad \mathrm{Co}_{3} \mathrm{O}_{4}, \quad \mathrm{Mn}_{3} \mathrm{O}_{4}$, among others-is an important group of compounds, and one that sees considerable use as electrode materials in lithium-ion batteries ${ }^{1-7}$. Specifically, magnetite $\left(\mathrm{Fe}_{3} \mathrm{O}_{4}\right)$ is a promising candidate as an anode material, as it is both inexpensive and non-toxic. Importantly, it also has a high electronic conductivity and can store up to eight $\mathrm{Li}$ ions per formula unit, which leads to a high theoretical specific capacity of $926 \mathrm{mAhg}^{-1}$ (refs 8-20). The structure of $\mathrm{Fe}_{3} \mathrm{O}_{4}$ is inverse spinel $\left(\left(\mathrm{Fe}^{3+}\right)_{8 \mathrm{a}}\right.$ $\left[\mathrm{Fe}^{2+} \mathrm{Fe}^{3+}\right]_{16 \mathrm{~d}} \mathrm{O}_{4}$, denoted by Wyckoff notation), where there are 64 tetrahedral sites in a unit cell (one-eighth of them are 8a sites occupied by $8 \mathrm{Fe}^{3+}$ cations) and 32 octahedral sites (half of them are $16 \mathrm{~d}$ sites occupied by $8 \mathrm{Fe}^{2+}$ and $8 \mathrm{Fe}^{3+}$ cations), leaving behind 56 empty tetrahedral ( $8 \mathrm{~b}$ and $48 \mathrm{f}$ ) sites and 16 empty octahedral (16c) sites in the interstitial space to allow for accommodation of guest $\mathrm{Li}^{+}$ions ${ }^{17-20}$. The lithiation of spinel iron oxide was investigated by Thackeray et al. ${ }^{16,17}$ using a combination of open-circuit voltage (OCV) measurements and $\mathrm{X}$-ray diffraction, and they posited that the overall lithiation of $\mathrm{Fe}_{3} \mathrm{O}_{4}$ follows the reaction equation shown in equation (1):

$$
8 \mathrm{Li}+\mathrm{Fe}_{3} \mathrm{O}_{4} \rightarrow 4 \mathrm{Li}_{2} \mathrm{O}+3 \mathrm{Fe}
$$

They suggested that during the initial stage of lithium insertion, the $\mathrm{Fe}^{3+}$ cations at tetragonal 8a sites are distorted into the nearby octahedral $16 \mathrm{c}$ sites by inserting $\mathrm{Li}^{+}$ions into the octahedral $16 \mathrm{c}$ sites, which leads to a lattice reconstruction of the spinel structure to form the rocksalt structure, that is, $\left(\mathrm{Li}^{+}{ }_{x} \mathrm{Fe}^{3+}\right)_{16 \mathrm{c}}\left[\mathrm{Fe}^{2+} \mathrm{Fe}^{3+}\right]_{16 \mathrm{~d}} \mathrm{O}_{4}$ (refs 16-21). This is an intercalation process, and thus it does not lead to a significant volume change. They proposed that further lithiation triggers destruction of the rocksalt structure along with an extrusion of metallic $\mathrm{Fe}$ from the material, which is commonly known as a conversion reaction. During the conversion reaction, the rocksalt structure transforms into a composite which is composed of both metallic $\mathrm{Fe}$ and $\mathrm{Li}_{2} \mathrm{O}$. Critically, this conversion leads to a drastic volume expansion $^{9-15}$.

$\mathrm{Fe}_{3} \mathrm{O}_{4}$ shows dramatic differences in electrochemical performance as a function of discharge rate ('C rate'), with the intercalation process not readily observed in the discharge voltage profiles $^{9-11}$. These differences cannot be understood from simple consideration of the reaction equation, which describes how these processes occur in an equilibrium condition. In one related example, the positive electrode material $\mathrm{LiFePO}_{4}$ (LFP) exhibits a solid-solution transformation at high rates when undergoing an intercalation reaction, specifically when the two-phase transition process is suppressed above a certain overpotential ${ }^{22,23}$. Thus, it is suggested that for $\mathrm{Fe}_{3} \mathrm{O}_{4}$ the overall charge-discharge performance depends strongly on the kinetics of specific discharge mechanisms. This in turn determines the realistic redox reaction characteristics that the battery sees in working conditions.

Finally, it is well-known that the rate capability of electrodes can be improved by reducing the material size from the micrometre to the nanometre regime. This improvement is due to both the increase in surface area and the reduction of electron/ion diffusion length ${ }^{24-26}$. However, in the case of nanoscale electrodes, the overall kinetics of lithiation is further complicated by local inhomogeneities that can occur in the electrochemical conditions. Besides the presence of both intercalation and conversion reactions, all of these facts underscore the importance of understanding the phase transitions of $\mathrm{Fe}_{3} \mathrm{O}_{4}$ that occur in non-equilibrium conditions, as it is these parameters that must be optimized when considering spinel-based electrode materials in electrochemical applications ${ }^{22}$.
In this work, we investigate how non-equilibrium intercalation and conversion reactions proceed by studying the lithiation process in real time. X-ray scattering or Mössbauer spectroscopy is most often used to identify the phase transformations that occur in nanosized electrode materials ${ }^{16,19}$, but these techniques have the disadvantage of providing only average information, and generally they lack the ability to probe reaction kinetics in real time. Critically, it is important to undertake lithiation studies in situ, as ex situ studies may provide inaccurate information due to changes that may occur during removal of the electrode materials from an actively lithiated condition ${ }^{22,27}$. In contrast, transmission electron microscopy (TEM) allows direct, real-time information to be obtained from structures at the atomic scale. The need for in situ observations of this type has been increasingly recognized, and thus there have been many recent reports of the use of in situ TEM techniques to study alloying and conversion reactions ${ }^{28-38}$. However, directly visualizing phase transitions that occur during intercalation reactions is challenging because the volume change that occurs during this type of reaction is negligible ${ }^{39-42}$. This prevents the use of standard diffraction contrast imaging techniques. In this work, we utilize a strain-sensitive, bright-field scanning TEM (BF-STEM) imaging technique to overcome this challenge. This approach allows us to observe the phase changes that occur in monodisperse $\mathrm{Fe}_{3} \mathrm{O}_{4}$ nanoparticles in situ during the intercalation reaction, and follow the subsequent conversion reaction directly. By integrating ex situ synchrotron X-ray and in situ TEM approaches, we capture in detail the mechanisms by which lithiation occurs and relate this directly to the reaction kinetics. Furthermore, we support these observations with both density functional theory (DFT) calculations and non-equilibrium phase-field theory to describe the atomistic processes from first principles and phenomenological perspective, respectively. This work highlights the importance of exploiting advanced TEM techniques to reveal reaction inhomogeneities at the nanoscale.

\section{Results}

Material structures and electrochemical properties. The as-synthesized $\mathrm{Fe}_{3} \mathrm{O}_{4}$ nanoparticles are $c a .80 \mathrm{~nm}$ truncated octahedrons, as shown in Fig. 1a and Supplementary Fig. 1. Atomic-resolution high-angle annular dark-field (HAADF) STEM images (as in Fig. 1b) indicate that the pristine $\mathrm{Fe}_{3} \mathrm{O}_{4}$ nanoparticles are single-crystalline with the inverse spinel structure ( $F d \overline{3} m$ space group) and terminated with $\{111\}$ crystal planes. To characterize the electrochemical performance, voltage profiles at different C-rates (1C, C/10 and C/200, or $926 \mathrm{~mA} \mathrm{~g}^{-1}$, $92.6 \mathrm{~mA} \mathrm{~g}^{-1}$ and $4.63 \mathrm{~mA} \mathrm{~g}^{-1}$, respectively) are shown in Fig. 1e. The first discharge capacity is $1,080 \mathrm{mAh}^{-1}$ at $\mathrm{C} / 200$, and it decreases to $916 \mathrm{mAhg}^{-1}$ and $914 \mathrm{mAhg}^{-1}$ for $\mathrm{C} / 10$ and $1 \mathrm{C}$, respectively, close to the theoretical capacity of $926 \mathrm{mAh} \mathrm{g}^{-1}$. The capacity excess in the initial discharge is generally believed due to side reactions involving electrolyte decomposition and formation of solid electrolyte interphases ${ }^{43-45}$, while the following cyclic tests show a rate performance (Supplementary Fig. 2) that is comparable to previous reports ${ }^{10-12}$. After full discharge (lithiation), the electrode materials have changed their structure and morphology dramatically to form a nanocomposite consisting of ultrafine $(<5 \mathrm{~nm}) \quad \mathrm{Fe}$ nanoparticles and an amorphous $\mathrm{Li}_{2} \mathrm{O}$ matrix phase (Fig. 1c), as confirmed by electron diffraction (Fig. 1d). It is noted that two distinct plateaus appear in the $\mathrm{C} / 200$ discharge curve, corresponding to the intercalation reaction and conversion reaction, respectively ${ }^{13-15,17}$, whereas the intercalation plateau is absent from the relatively faster discharge curves at rates of $1 \mathrm{C}$ and $\mathrm{C} / 10$. This can be due to enhancements in the reaction 

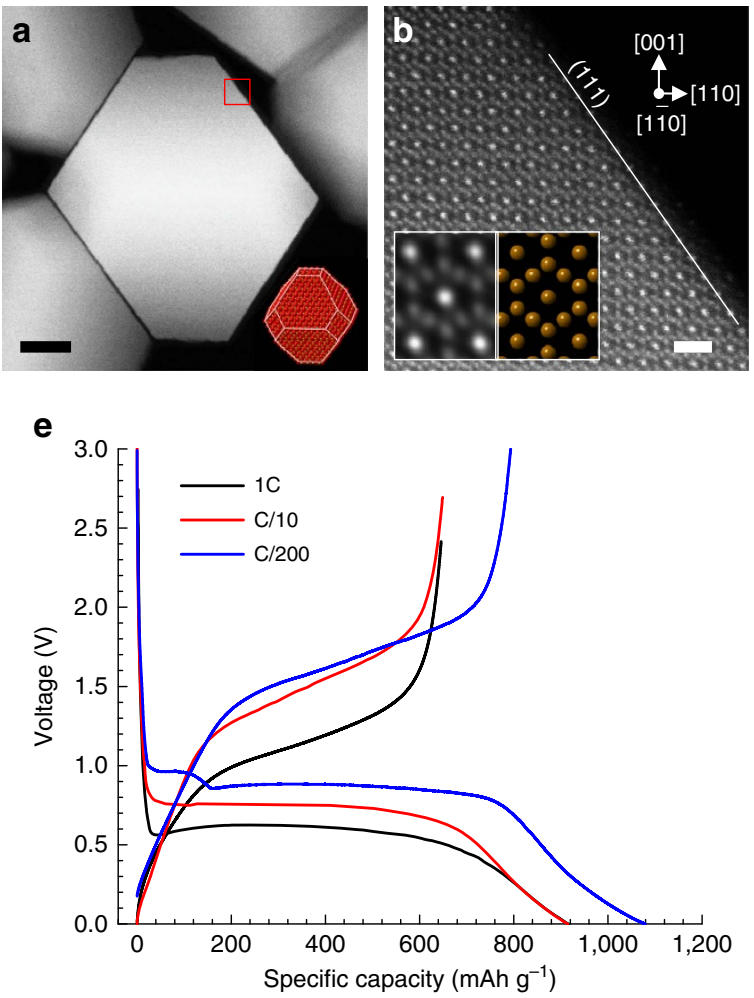

g

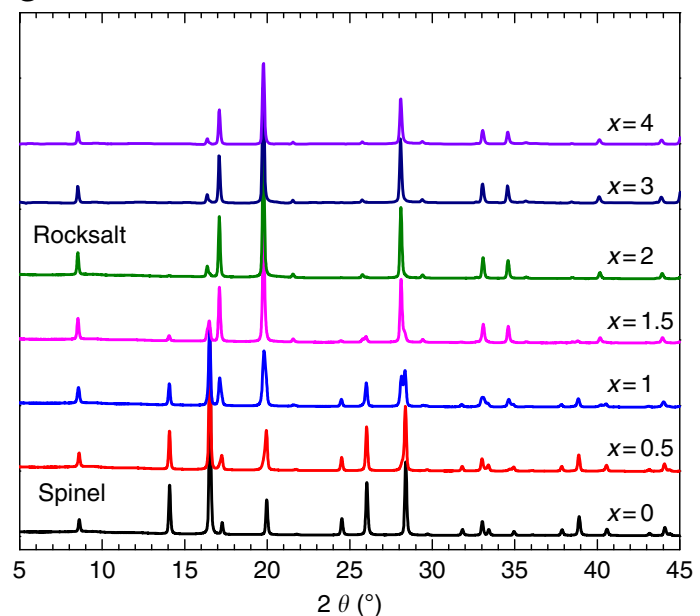

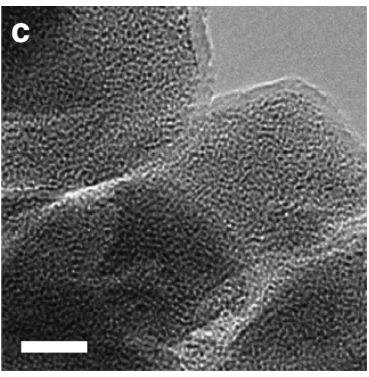

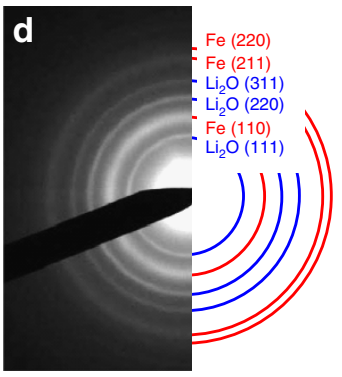

f

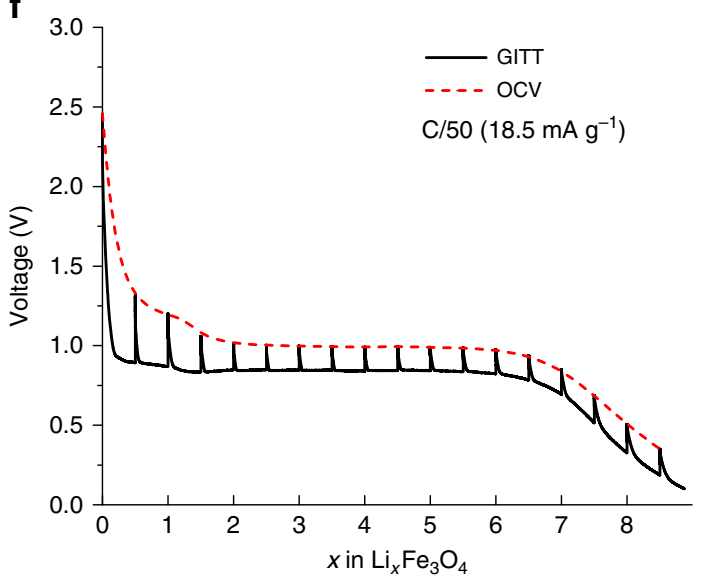

$\mathbf{h}$

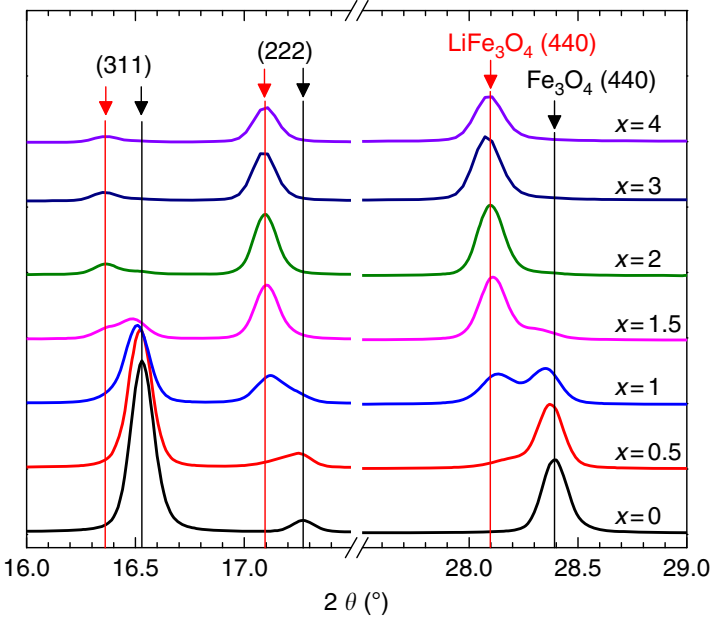

Figure 1 | Electrode materials and electrochemical performance. (a) STEM image of the pristine $\mathrm{Fe}_{3} \mathrm{O}_{4}$ single crystals. Inset showing the crystals in the truncated octahedral shape. (b) High-resolution HAADF-STEM image showing spinel structure along [1-10] zone axis. Inset showing enlarged STEM image compared with the atom model. (c) TEM image and (d) SAED pattern of the fully lithiated electrode, confirming the nanocomposite of Fe nanocrystals in amorphous $\mathrm{Li}_{2} \mathrm{O}$ matrix. Scale bars, (a,c) $20 \mathrm{~nm} ;(\mathbf{b}) 1 \mathrm{~nm}$. (e) First-cycle charge/discharge profiles of coin-cell batteries at rates of $1 \mathrm{C}, \mathrm{C} / 10$ and $\mathrm{C} / 200$ (that is, $926 \mathrm{mAg}^{-1}, 92.6 \mathrm{mAg}^{-1}$ and $4.63 \mathrm{mAg}^{-1}$ ). (f) GITT and OCV profiles in the first discharge cycle measured at C/50 (18.5 mA g ${ }^{-1}$ ). GITT measurements were performed by applying an intermittent current of $\mathrm{C} / 50$ for $3.125 \mathrm{~h}$ followed by a $24 \mathrm{~h}$ relaxation period. (g) SXRD patterns of $\mathrm{Li}_{x} \mathrm{Fe}_{3} \mathrm{O}_{4}$ at various SOCs $(x=0,0.5,1,1.5,2,3,4)$. (h) Enlarged SXRD patterns showing detailed peak shifts of (311), (222) and (440) reflections. Fe $\mathrm{O}_{4}$ and $\mathrm{LiFe}_{3} \mathrm{O}_{4}$ phases are marked by black and red arrows. X-ray wavelength is $0.72768 \AA$.

kinetics that can occur at high discharge current densities (such as voltage polarization). To justify this hypothesis, we employed the galvanostatic intermittent titration technique (GITT) ${ }^{46}$ for a discharge cycle at $\mathrm{C} / 10$ to measure the OCV profile: this reflects the equilibrium redox potentials at different states of charge (SOCs). Figure if clearly indicates the voltage polarization between the OCV and regular discharge profile, and confirms the existence of an intercalation plateau which is related to the charge rate. The intercalation process can generally be expressed as equation (2):

$$
x \mathrm{Li}+\mathrm{Fe}_{3} \mathrm{O}_{4}(\text { spinel }) \rightarrow \mathrm{Li}_{x} \mathrm{Fe}_{3} \mathrm{O}_{4} \text { (rocksalt) }
$$

where $x$ is the lithium content per formula. For an intercalation reaction, $x$ is supposedly between 0 and 2 (refs 16-18), and its upper limit depends on the total amount of lithium ions accommodated at the tetragonal $8 \mathrm{a}$ sites ${ }^{16,17}$. Further lithiation would trigger the conversion reaction. This is also confirmed by high-resolution TEM (HRTEM) imaging of ex situ lithiated 
$\mathrm{Li}_{x} \mathrm{Fe}_{3} \mathrm{O}_{4}$ with Li content $x=2,3,4,5,6,7,8$ (Supplementary Fig. 3). These HRTEM images and selected area electron diffraction (SAED) patterns show the transition from rocksalt $\mathrm{Li}_{x} \mathrm{Fe}_{3} \mathrm{O}_{4}$ to $\mathrm{Fe}+\mathrm{Li}_{2} \mathrm{O}$ composite in a gradually proceeding manner. Since the intercalation reaction only involves Li content $x<2$, it is crucial to focus our study on the first half of the lithiation. To study the intercalation process further, we utilized synchrotron X-ray diffraction (SXRD) to identify the phase changes at various SOCs of $x=0,0.5,1,1.5,2,3$ and 4 , as shown in Fig. 1g,h. The series of SXRD patterns show that the electrode materials transfer from spinel structure to rocksalt structure with increasing $\mathrm{Li}$ composition (detailed indexing is presented in Supplementary Fig. 4). From the enlarged patterns in Fig. 1h, we indeed identified the two stable $\mathrm{Fe}_{3} \mathrm{O}_{4}$ and $\mathrm{LiFe}_{3} \mathrm{O}_{4}$ phases. The nanosized $\mathrm{Fe}$ phase that resulted from the conversion reaction cannot be captured by SXRD even up to $x=4$; however, its presence was confirmed using TEM (Supplementary Fig. 5). These observations clearly indicate that SXRD—due to its lack of sensitivity in detecting nanoscale particles, and its averaging of the overall phase information-is not able to precisely determine either the initiation of the conversion reaction or to follow its evolution. In addition, the absence of an intercalation plateau in the cycling profiles of $1 \mathrm{C}$ and $\mathrm{C} / 10$ implies that the intercalation process is bypassed at high rates. Although the SXRD results clearly show the existence of a rocksalt intermediate phase, which arises from the intercalation reaction, the presence of this phase might also be due to a possible structure relaxation and phase separation that occurs post-mortem. Effects of this type have been reported in LFP system, where ex situ characterization is shown to inaccurately describe the reaction processes that are occurring ${ }^{21}$. In addition, the intercalation phase transition can either follow a two-phase model or solid-solution process depending on the details of the reaction kinetics, a process which cannot be resolved from the ex situ study. To solve these issues, we have utilized in situ electron microscopy approaches to determine which reaction pathways occur in spinel oxide.

Phase evolution by in situ electron diffraction. We utilized a dry cell set-up for our in situ TEM investigation ${ }^{36-38}$. This approach can precisely track the phase evolution at very high spatial resolution. Since there are no liquid organic electrolyte involved in the reactions, to verify the consistency in the phase transitions between the ex situ and in situ experiments, we tracked the dynamical phase evolution using in situ electron diffraction throughout the entire lithiation process, as shown in Fig. 2a and Supplementary Movie 1 The radially integrated intensity profiles from a series of timesequenced SAED patterns are plotted as a function of lithiation time, with the intensity represented by false colours (Fig. 2a). It is obvious that both the position and intensity of the Bragg reflections change as the lithiation proceeds, verifying the overall phase transformation from pristine $\mathrm{Fe}_{3} \mathrm{O}_{4}$ to the eventual $\mathrm{Fe}$ and $\mathrm{Li}_{2} \mathrm{O}$ composite (and in the same manner as the ex situ results). Using the intensity profiles of $\mathrm{Fe}_{3} \mathrm{O}_{4}$ (311) and $\mathrm{Fe}$ (110) SAED peaks as a measure, the gradual evolution of the phase transformation becomes clear, as depicted in Fig. 2b. It is also worth noting that the Bragg peaks of the $\mathrm{Fe}_{3} \mathrm{O}_{4}$ phase display a negative shift in reciprocal space (as indicated by arrows in Fig. 2a) during the initial stage of lithiation (up to $\sim 1,000 \mathrm{~s}$ ), which corresponds to an increase in the lattice parameter as $\mathrm{Li}^{+}$ions are inserted into the $\mathrm{Fe}_{3} \mathrm{O}_{4}$ lattice (Fig. 2c). This lattice parameter increase is quantitatively consistent with the phase transition from $\mathrm{Fe}_{3} \mathrm{O}_{4}$ to $\mathrm{LiFe}_{3} \mathrm{O}_{4}$, as extracted from our SXRD measurements. After $\sim 1,000 \mathrm{~s}$, the lattice expansion a

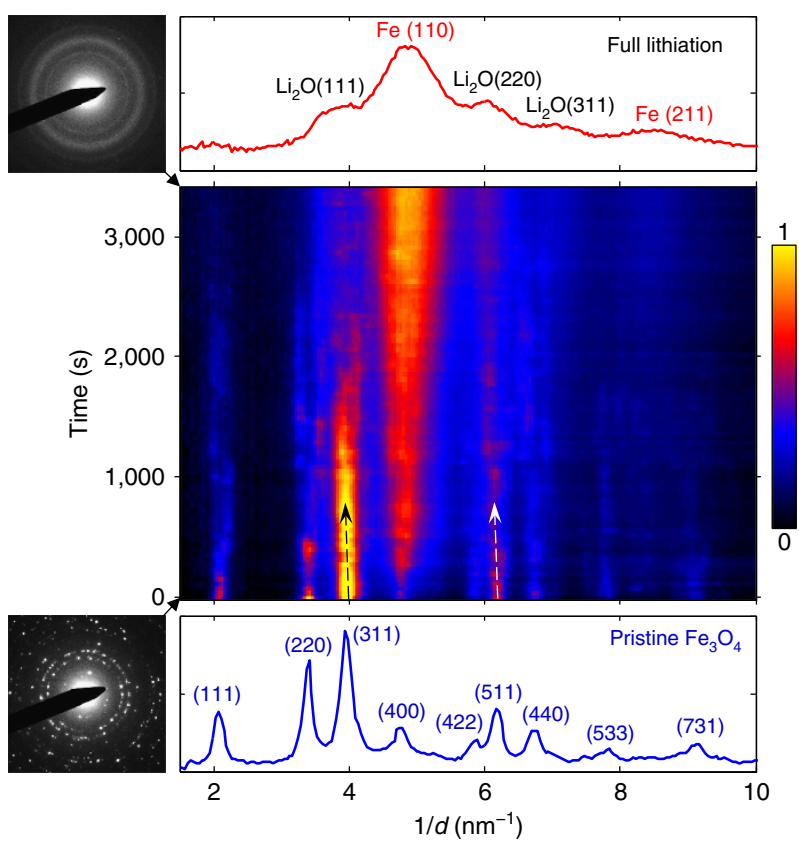

b
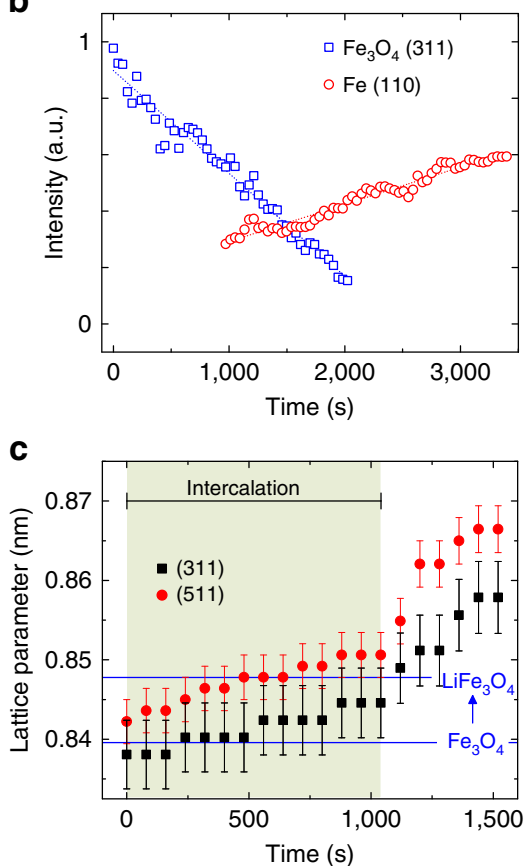

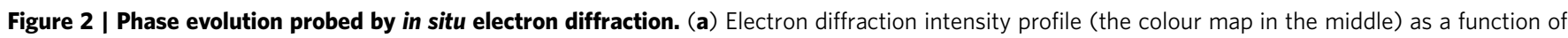
reaction time during an in situ lithiation of $\mathrm{Fe}_{3} \mathrm{O}_{4}$ nanoparticles (see Supplementary Movie 1). The SAED patterns and corresponding radially integrated intensity profiles obtained at pristine $(\mathrm{Os})$ and fully lithiated (3400 s) states are shown below and above the colour map, respectively. Arrows indicate diffraction peak shift towards a lower value in the reciprocal space. The sample was lithiated at a rate of $\sim 980 \mathrm{~mA} g-1$. (b) Intensity profiles of Fe $\mathrm{O}_{4}$ ( 311 ) and $\mathrm{Fe}(110)$ Bragg reflections during the lithiation, indicating the phase evolution of $\mathrm{Fe}_{3} \mathrm{O}_{4}$ and Fe. (c) Lattice parameter measured from Fe $\mathrm{O}_{4}$ (311) and (511) reflections showing an incremental shift as a function of lithiation time, which quantitatively agrees with the SXRD results. Error bars are given by 1 pixel precision of SAED measurements. Blue lines indicate lattice parameters of $\mathrm{Fe}_{3} \mathrm{O}_{4}$ and $\mathrm{LiFe}_{3} \mathrm{O}_{4}$ measured by SXRD in Fig. $1 \mathrm{~g}$. 
becomes more severe, indicating that the subsequent lithiation was dominated by the conversion reaction, as per equation (3).

$$
(8-x) \mathrm{Li}+\mathrm{Li}_{x} \mathrm{Fe}_{3} \mathrm{O}_{4} \rightarrow 4 \mathrm{Li}_{2} \mathrm{O}+3 \mathrm{Fe}
$$

Visualization of two-step lithiation by in situ BF-STEM. After verifying that the phase transformation occurs using in situ SAED, we performed an in situ BF-STEM observation to follow how the structure evolves during the entire lithiation process in real space, as shown in Fig. 3a and Supplementary Movie 2. The time-sequenced BF-STEM images record the intensity of the transmitted electrons scattered to lower angles, as well as the direct beam (involving the most coherent electron scattering). This signal is strain sensitive and thus enables direct visualization of the small lattice changes that occur during the intercalation process (Supplementary Fig. 6$)^{47}$. We show that this is essential observing intercalation reactions in real time. This is in contrast to other, more conventional imaging techniques (such as HAADF-STEM or BF/DF TEM) which do not have the requisite image sensitivity to directly observe this process (sensitivity to strain is not satisfied due to incoherent contrast or bending contour). The first intercalation step follows the reaction described in equation (2) to generate the lithiuminserted $\mathrm{Li}_{x} \mathrm{Fe}_{3} \mathrm{O}_{4}$ phase (shown with a lighter contrast). These regions will be further lithiated in the subsequent conversion reaction, which is also accompanied by the extrusion of ultrafine metallic $\mathrm{Fe}$ nanoparticles to form a composite with the amorphous $\mathrm{Li}_{2} \mathrm{O}$. For better visualization, we use false colours to distinguish the pristine $\mathrm{Fe}_{3} \mathrm{O}_{4}$ (red), Li-inserted $\mathrm{Fe}_{3} \mathrm{O}_{4}$ (blue) and completely conversion composite (green) phases and their evolution as a function of time. This was also verified using electron energy-loss spectroscopy (EELS) in STEM (Supplementary Fig. 7). We note that, under realistic electrochemical environment, the lithiation process shows a non-equilibrium reaction pathway, that is, the conversion reaction starts to take place before the intercalation completely finishes. This may be likely due to the fact that the diffusion of lithium on the $\mathrm{Fe}_{3} \mathrm{O}_{4}$ particle surface is faster than that through the lithiated $\mathrm{Li}_{x} \mathrm{Fe}_{3} \mathrm{O}_{4}$ phase, which can cause a sufficiently large lithium concentration at the particle surface and thereby trigger the conversion reaction to happen at an early stage (but still after the intercalation reaction). It is obvious that both intercalation and conversion reactions follow a 'shrinking-core' mode, proceeding from the outer surface to the inner region ${ }^{37}$. Specifically, the initial intercalation trajectory does not exhibit any preferential directions, in accord with the zigzag $\mathrm{Li}^{+}$diffusion paths in three-dimensional (3D) tunnels ${ }^{16}$, whereas the subsequent conversion tends to propagate through the outer facets, that is, $\{111\}$ planes. As an approximate measure of reaction kinetics, we quantified the projected areas of the three phases versus the lithiation time, displayed in Fig. 3b. This figure shows that the propagation speed of the intercalation process is about 1 order of magnitude faster than the following conversion reaction (Fig. 3c). We note that these measurements are from one single particle, where the electrochemical conditions are supposed to be identical. This is consistent with the fact that full conversion needs multiple Li-ion transfers, when compared with the intercalation process. We do not believe this to be an effect resulting from local electrochemical inhomogeneity.

HRTEM characterization. For a fundamental understanding of the intercalated phase, we conducted HRTEM imaging to track the atomic structure changes for both in situ and ex situ scenarios, as shown in Fig. 4. A new phase with the rocksalt structure was observed in situ as $\mathrm{Li}^{+}$ions were inserted into the spinel $\mathrm{Fe}_{3} \mathrm{O}_{4}$ phase (Fig. 4a,b). Using two sets of diffraction spots from the fast Fourier transform (FFT, Fig. 4a insets), we can separately map the distribution of the spinel (red) and rocksalt (green) phases and thus visualize the propagation of the intercalation front in the
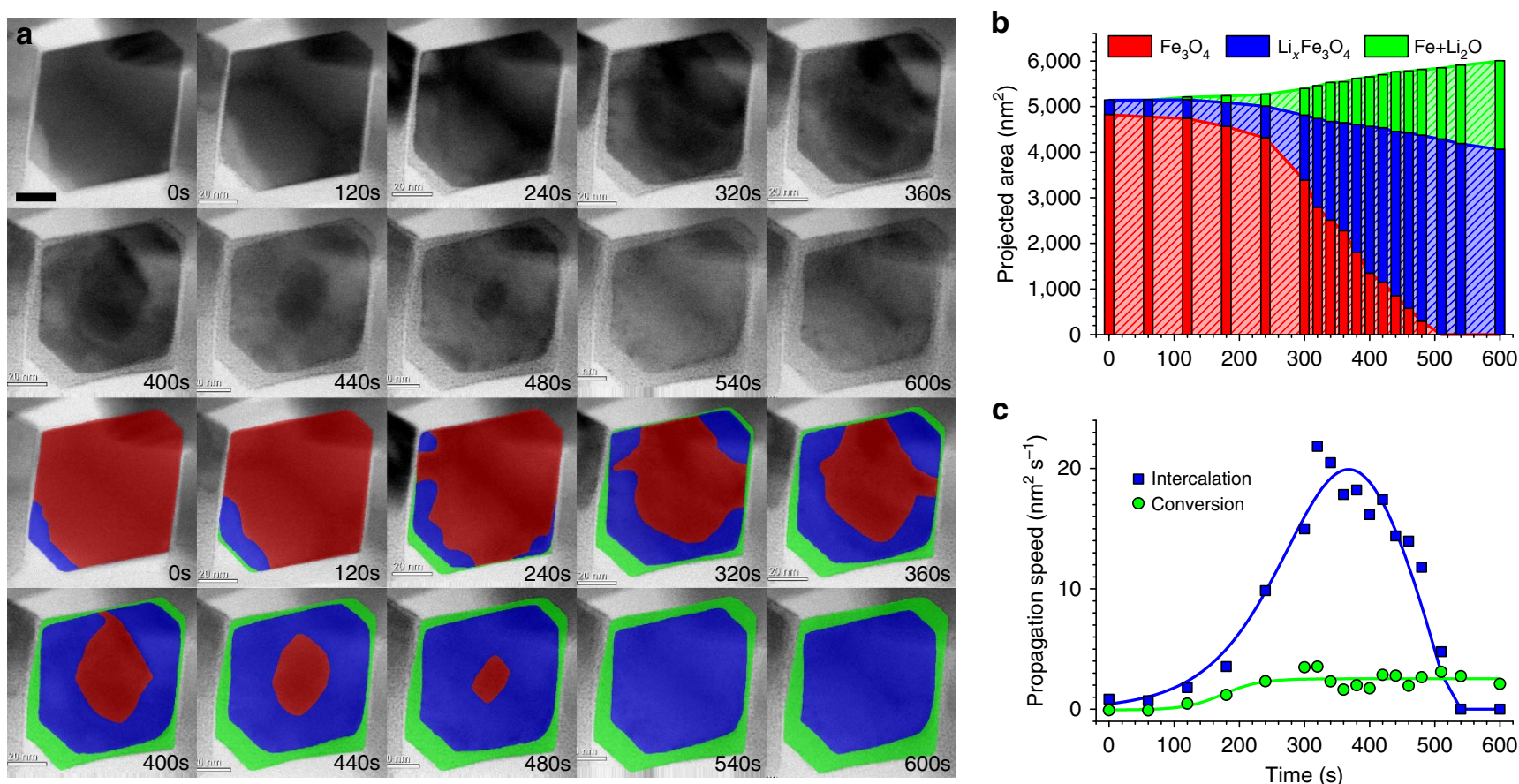

C

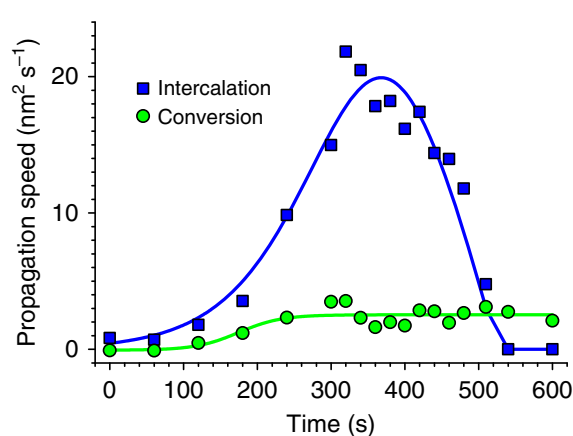

Figure 3 | In situ observation of two-step phase transformation during lithiation. (a) BF-STEM image series showing phase evolution during lithiation The overlaid false colours indicate different phases: pristine $\mathrm{Fe}_{3} \mathrm{O}_{4}$ (red), Li-inserted $\mathrm{Li}_{x} \mathrm{Fe}_{3} \mathrm{O}_{4}$ (blue) and $\mathrm{Fe}+\mathrm{Li}_{2} \mathrm{O}$ composite after conversion (green). Scale bar, $20 \mathrm{~nm}$. The sample was lithiated at a rate of $\sim 2,700 \mathrm{mAg}^{-1}$. (b) Projected areas of the three phases as a function of time. (c) Propagation speed of intercalation and conversion as a function of time. 

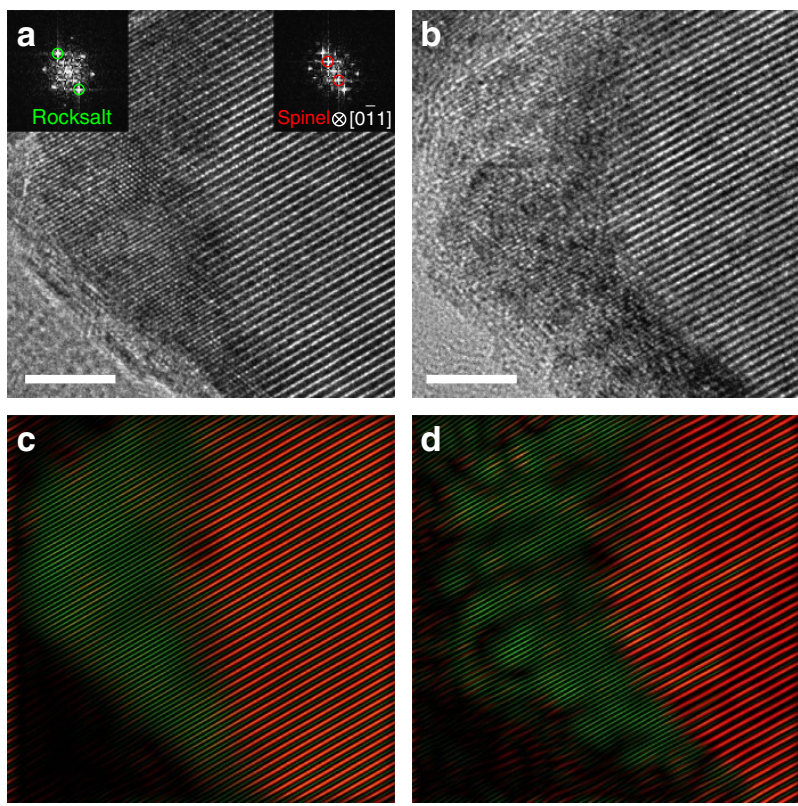
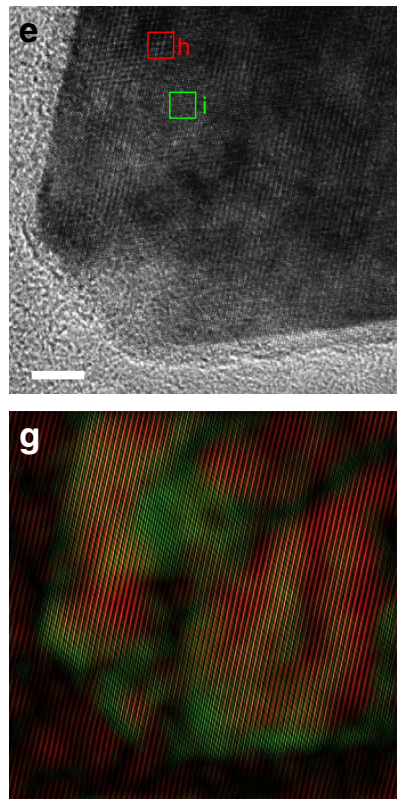
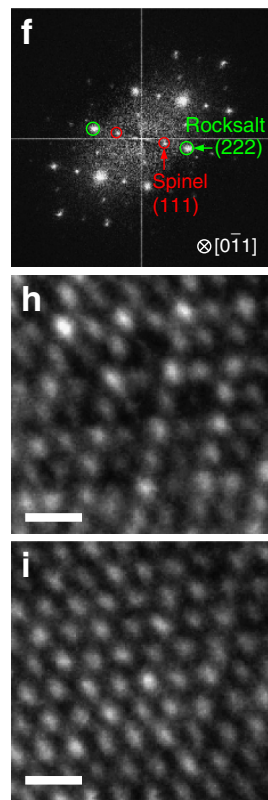

Figure 4 | Atomic structural evolution of phase transformation. (a,b) HRTEM images of a $\mathrm{Fe}_{3} \mathrm{O}_{4}$ single crystal showing the spinel and rocksalt phases during in situ lithium intercalation. Insets showing the FFT of the spinel (top-right of panel) and rocksalt (top-left of panel) structures along [0-11] zone axis. (e) HRTEM image of a partially lithiated $\mathrm{Fe}_{3} \mathrm{O}_{4}$ single crystal from ex situ lithiation. (f) The FFT of e. (h,i) The enlarged HRTEM images of boxed regions in e showing spinel and rocksalt structures, respectively. (c,d,g) Filtered images of $(\mathbf{a}, \mathbf{b}, \mathbf{e})$ using two sets of spinel (red) and rocksalt (green) diffraction spots to indicate the corresponding phase distribution. The samples were lithiated at $\sim 150 \mathrm{mAg}^{-1}$ for in situ lithiation, and $92.6 \mathrm{mAg}{ }^{-1}$ for ex situ lithiation. Scale bars: $(\mathbf{a}, \mathbf{b}, \mathbf{e}) 5 \mathrm{~nm} ;(\mathbf{h}, \mathbf{i}) 5 \AA$.

reacting $\mathrm{Fe}_{3} \mathrm{O}_{4}$ crystal (Fig. 4c,d). Similarly, in an ex situ lithiated sample that was discharged to $0.9 \mathrm{~V}$, we reproduced this result, and found a similar mixture of spinel and rocksalt phases (Fig. $4 \mathrm{e}, \mathrm{g}$ ). In the pristine spinel $\mathrm{Fe}_{3} \mathrm{O}_{4}$ crystals, Fe cations occupy the tetrahedral $8 \mathrm{a}$ sites and octahedral $16 \mathrm{~d}$ sites, which give the uneven TEM contrast (dim at the diamond centre) shown in Fig. 4h. Upon lithium insertion, all the cations migrate to the octahedral sites (16c and 16d), leading to a uniform contrast (Fig. 4i). This means that the inserted Li ions occupy the 16c sites and also repel the adjacent Fe cations from the $8 \mathrm{a}$ to the $16 \mathrm{c}$ sites to form $\mathrm{Li}_{x} \mathrm{Fe}_{3} \mathrm{O}_{4}$ crystals with the rocksalt structure, with lithium composition up to $x=1$. It is also noted from previous reports that the excess Li ions (up to $x=2$ ) could possibly insert into the $8 \mathrm{~b}$ and $48 \mathrm{f}$ sites to form $\mathrm{Li}_{2} \mathrm{Fe}_{3} \mathrm{O}_{4}$ phase without Fe extrusion ${ }^{16,17}$. However, this particular phase was not experimentally identified from TEM observations. Nevertheless, the intercalation process goes through a two-phase mode and forms an intermediate phase of $\mathrm{Li}_{x} \mathrm{Fe}_{3} \mathrm{O}_{4}$ (refs 16-18). Thereafter, the conversion reaction occurs and the $\mathrm{Li}_{x} \mathrm{Fe}_{3} \mathrm{O}_{4}$ phase is decomposed into $\mathrm{Li}_{2} \mathrm{O}$ and metallic Fe nanocrystals, which can be observed as the speckles in Fig. 4b,d. Combining both in situ TEM imaging and ex situ SXRD results, we now can conclude that the intercalation proceeds in a two-phase 'nucleation and growth' manner, resulting in sharp interfacial boundaries between the spinel and rocksalt phases, as expressed in equation (2).

First principles calculations and phase-field modelling. DFT computation has been performed to calculate the voltage profile of the lithiation process under the thermodynamic equilibrium condition $^{48-52}$. The calculated reaction pathway has multiple voltage plateaus (green dashed curve in Fig. 5a) with intermediate phases, such as $\mathrm{LiFeO}_{2}, \mathrm{Li}_{2} \mathrm{FeO}_{2}$ and $\mathrm{Li}_{5} \mathrm{FeO}_{4}$ (Fig. 5b). Given that the $\mathrm{LiFe}_{3} \mathrm{O}_{4}$ is an intermediate phase experimentally observed from SXRD, our DFT calculations also considered a two-step reaction route (red curve in Fig. 5a), including the intercalation plateau $\left(\mathrm{Li}+\mathrm{Fe}_{3} \mathrm{O}_{4} \rightarrow \mathrm{LiFe}_{3} \mathrm{O}_{4}\right)$ and the conversion plateau
$\left(\mathrm{LiFe}_{3} \mathrm{O}_{4} \rightarrow \mathrm{Li}_{2} \mathrm{O}+\mathrm{Fe}\right)$. The ground-state $\mathrm{LiFe}_{3} \mathrm{O}_{4}$ structure as illustrated in the inset crystal models of Fig. 5a possesses a similar structural framework (that is, $[\mathrm{Fe}]_{16 \mathrm{~d}} \mathrm{O}_{2}$ octahedron) that was inherited from the spinel $\mathrm{Fe}_{3} \mathrm{O}_{4}$ structure. Consistent with the ground-state $\mathrm{LiFe}_{3} \mathrm{O}_{4}$ structure calculated in the DFT computation, $\mathrm{Li}$ ions insert into the octahedral $16 \mathrm{c}$ openings, and repel the nearby $8 \mathrm{a}$ Fe ions to the other surface-sharing $16 \mathrm{c}$ site, which forms the structure identical to that observed in the HRTEM images in Fig. 4. In addition, since the octahedral 16c sites are interconnected as a $3 \mathrm{D}$ channel, the initial intercalation would proceed in a disordered pattern, which explains the irregular patterns that are observed during the shrinking-core process in Fig. 3. After all of the octahedral 16c sites are filled by $\mathrm{Li}$, the rocksalt lattice becomes more isotropic to the incoming $\mathrm{Li}$ ions, which makes the subsequent conversion most likely to propagate across the outline of crystal, again as observed in Fig. 3.

\section{Discussion}

Given the basics of chemical thermodynamics, the equilibrium lithiation should go through the $\mathrm{LiFe}_{3} \mathrm{O}_{4}$ phase and the subsequent $\mathrm{Fe}+\mathrm{Li}_{2} \mathrm{O}$ composite in two non-overlapping processes, as proposed by Thackeray et al. ${ }^{16-18}$. However, our in situ results show a clear overlap between the two steps, suggesting that the kinetic effects play an important role during lithiation. On the other hand, the phase-field theory, taking into account the effect of overpotentials, has succeeded in explaining the electrochemical kinetics in lithium-ion battery systems ${ }^{53-55}$. We performed the phase-field simulation in the frame of non-equilibrium thermodynamics based on the Butler-Volmer equation $^{55,56}$ and the Cahn-Hilliard equation ${ }^{23,57}$. To simulate the two-step reaction, we constructed a homogeneous free-energy function with three local minima corresponding to the pristine, intercalation and conversion phases, respectively. In addition, the kinetic contributions from overpotential and volumetric strain 

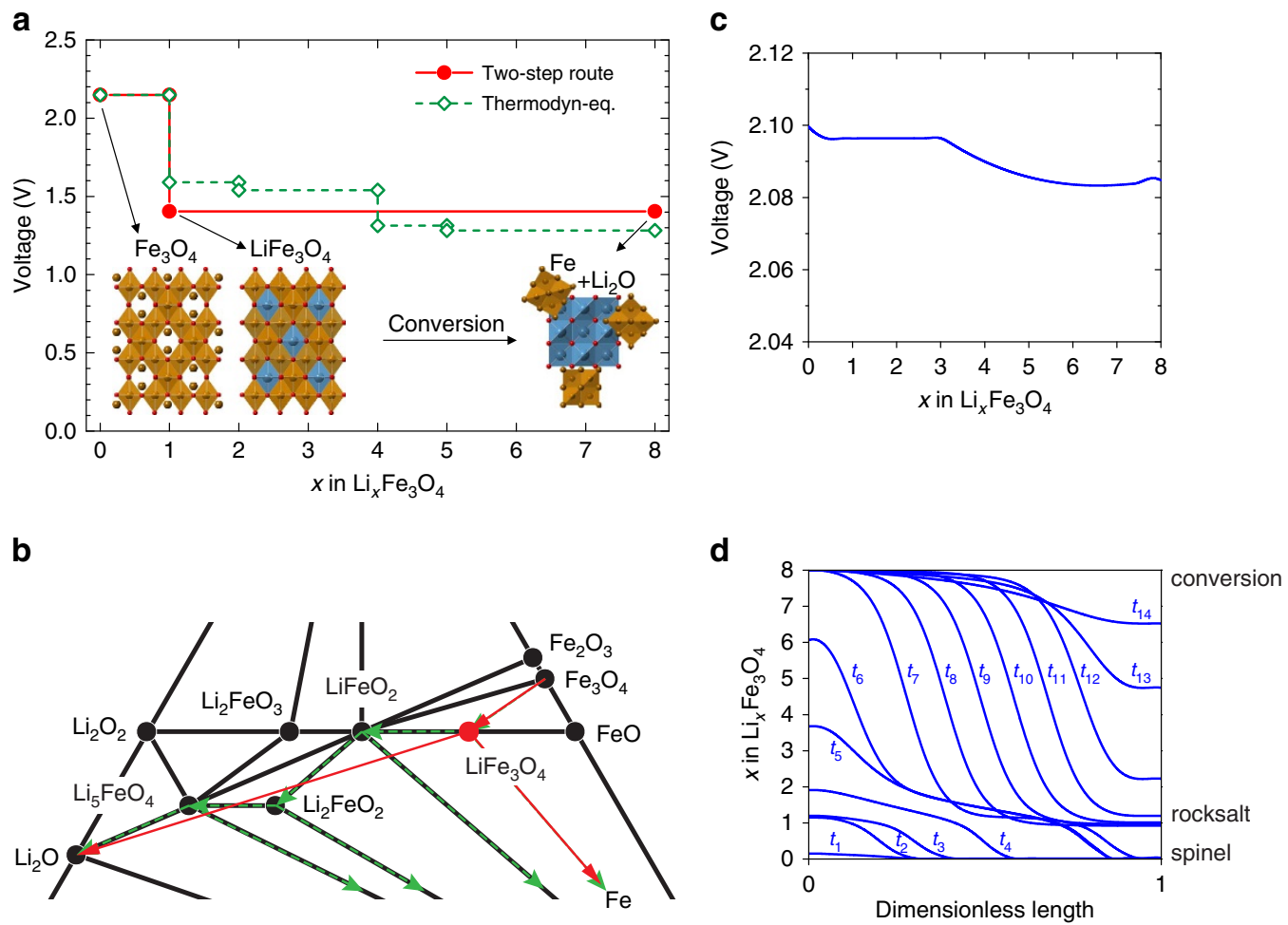

Figure 5 | DFT calculation and phase-field simulation of lithiation process in $\mathbf{F e}_{\mathbf{3}} \mathbf{O}_{\mathbf{4}}$ crystals. (a) The voltage profiles as a function of $\mathrm{Li}$ composition $(x=0-8)$ calculated by DFT showing both thermodynamic equilibrium (green dashed lines) and two-step reaction (red solid lines) routes for an entire lithiation process. The inset models showing crystal structures of $\mathrm{Fe}_{3} \mathrm{O}_{4}$ and $\mathrm{LiFe}_{3} \mathrm{O}_{4}$ phases during the intercalation process and a schematic of $\mathrm{Li}_{2} \mathrm{O}$ plus metallic Fe after the complete conversion reaction. Colour spheres representing Fe (yellow), Li (blue) and O (red). (b) Li-Fe-O phase diagram with overlaid arrow lines showing lithiation pathways through thermodynamic equilibrium multistep route (green dashed lines) or experimentally observed two-step route (red solid lines). (c) Discharge voltage profiles calculated from the phase-field modelling. (d) Li composition profiles along one-dimensional length as a function of reaction time simulated by the phase-field theory (also see Supplementary Movie 3). The time intervals are uniform between adjacent time stamps.

have also been included (details in Supplementary Methods). The calculated discharge voltage profile is shown in Fig. 5c, which qualitatively agrees with the experimental curves in Fig. 1e. Figure $5 \mathrm{~d}$ shows the calculated lithium compositional profiles as a function of time ( $x$-axis is the dimensionless lithiation direction and $y$-axis is the Li composition, details shown in Supplementary Movie 3). It is found that the formation of $\mathrm{LiFe}_{3} \mathrm{O}_{4}$ phase is predominant in the early stage of lithiation, whereas the conversion reaction initiates immediately afterward and propagates before the complete intercalation, resulting in a mixed lithiation behaviour, which is in good agreement with the in situ STEM observation (Fig. 3a). It is well-known that the lithiation process (redox stage and reaction speed) in a real battery is heterogeneous due to the fluctuation of local environments ${ }^{22}$. Here we reasonably assume that the local electrochemical conditions are identical for these two reactions within one nanoparticle. By comparing the in situ STEM results with phase-field simulation, we believe that the coexistence of $\mathrm{Fe}_{3} \mathrm{O}_{4}, \mathrm{Li}_{x} \mathrm{Fe}_{3} \mathrm{O}_{4}$, and $\mathrm{Fe}+\mathrm{Li}_{2} \mathrm{O}$ phases are due to the competition between the intercalation and conversion reactions. The overall electrochemical kinetics dictates to the applied C-rate, which in turn determines the propagation speeds of the interfaces of $\mathrm{Fe}_{3} \mathrm{O}_{4} / \mathrm{Li}_{x} \mathrm{Fe}_{3} \mathrm{O}_{4}$ and $\mathrm{Li}_{x} \mathrm{Fe}_{3} \mathrm{O}_{4} / \mathrm{Fe}+\mathrm{Li}_{2} \mathrm{O}$. Although the reaction speed of intercalation is much faster, the conversion reaction accommodates more Li ions. Therefore, in terms of total lithium insertion capacity, both reactions give important contributions to the overall energy storage rate.

The kinetic lithiation mechanism we proposed here can accommodate the following phenomena which could not be interpreted by the equilibrium theory. (1) As for the discharge profiles in Fig. 1e and in literature ${ }^{9-11}$, even the intercalation reaction has not completed, the occurrence of conversion reaction on the surface will reduce the apparent discharge voltage, which consequently flattens the first plateau. (2) As for the previous debate on the existence of metallic Fe extrusion ${ }^{16-19}$, due to the kinetic effect, the conversion reaction can happen at the same time of the intercalation reaction, which induces the formation of metallic Fe. For the discharge process at a low rate, the extrusion of metallic $\mathrm{Fe}$ is not expected to be observed.

In summary, we have investigated the lithiation mechanism of spinel magnetite using an in situ strain-sensitive, BF-STEM technique, as well as DFT computation and phase-field simulation. By explicit visualization of the two-step intercalationconversion process of the lithiation in $\mathrm{Fe}_{3} \mathrm{O}_{4}$ nanocrystals, we found that the initial lithium intercalation leads to formation of the rocksalt $\mathrm{LiFe}_{3} \mathrm{O}_{4}$ phase in a two-phase reaction mode, and the lithium intercalation process significantly overlaps with the subsequent conversion reaction within a single nanoparticle, leading to indistinctness of the discharge profiles. This scenario aslo clarifies the mechanism of metallic $\mathrm{Fe}$ extrusion during the intercalation process. Our findings have elucidated the ionic occupancy on the atomic level and revealed how rate-dependent kinetic effects can affect the reaction pathway at the singleparticle scale. These findings highlight the importance of advanced in situ electron microscopy techniques in the field of lithium-ion batteries and provide valuable insights to improve the electrochemical performances of other spinel lithium metal oxide cathode materials. 


\section{Methods}

Materials. Iron acetylacetonate $\left(\mathrm{Fe}(\mathrm{acac})_{3}, 99 \%\right)$, oleic acid (OAc, 90\%), benzyl ether $(\mathrm{BE}, 99 \%)$ and nitrosonium tetrafluoroborate $\left(\mathrm{NOBF}_{4}, 95 \%\right)$ were purchased from Sigma-Aldrich and used without any further purification. All solvents, including hexane, isopropanol, toluene and dimethylformamide (DMF), were in ACS reagent grade and were purchased from Fisher Scientific and used without further purification. Nanoparticle synthesis was performed using standard Schlenk techniques.

\section{Sample preparation. The $\mathrm{Fe}_{3} \mathrm{O}_{4}$ nanoparticles were synthesized by the} decomposition of $\mathrm{Fe}(\mathrm{acac})_{3}$ in the presence of $\mathrm{OAc}$, as modified from the approach in ref 58. In a typical synthesis, $2 \mathrm{mmol}$ of $\mathrm{Fe}(\mathrm{acac})_{3}$ was mixed with $1.28 \mathrm{ml}$ of OAc and $10 \mathrm{ml}$ of BE. The mixture was magnetically stirred and was kept under vacuum at $100{ }^{\circ} \mathrm{C}$ to generate a dark red solution. After evacuation at $100^{\circ} \mathrm{C}$ for $1 \mathrm{~h}$ to remove all impurities and moisture, the system was filled with $\mathrm{N}_{2}$ and the solution was further heated to $290^{\circ} \mathrm{C}$ at a rate of about $15^{\circ} \mathrm{C} \mathrm{min}-1$ and kept at this temperature for $20 \mathrm{~min}$. After cooling to room temperature, the NPs were separated by adding isopropanol, followed by centrifugation (8,500 r.p.m., $5 \mathrm{~min}$ ). The NPs were further purified by sequential operations of dispersing in hexane, precipitation by adding isopropanol and centrifugation. The as-synthesized $\mathrm{Fe}_{3} \mathrm{O}_{4}$ nanoparticles were surface-passivated by $\mathrm{OAc}$, which can be ligand-exchanged by $\mathrm{NOBF}_{4}$ according to literature ${ }^{59}$. About $0.1 \mathrm{~g}$ of $\mathrm{NOBF}_{4}$ was added into $10 \mathrm{ml} \mathrm{DMF}$, resulting in immediately a light green solution. This $\mathrm{NOBF}_{4} \mathrm{DMF}$ solution is added to the hexanes solution $(10 \mathrm{ml})$ of $\mathrm{Fe}_{3} \mathrm{O}_{4} \mathrm{NPs}(100 \mathrm{mg})$. The mixture was shaken for $10 \mathrm{~min}$, followed by the addition of $25 \mathrm{ml}$ of toluene. The $\mathrm{NOBF}_{4}$-modified $\mathrm{Fe}_{3} \mathrm{O}_{4}$ NPs were collected through centrifugation (7,000 r.p.m., 8 min). The $\mathrm{Fe}_{3} \mathrm{O}_{4} \mathrm{NPs}$ after ligand exchange can be solubilized in DMF with sonication. To remove residual organics, the NPs were further purified by two cycles of re-dispersing in DMF and precipitation by adding toluene centrifugation. The NPs were either stored in DMF or evacuated at the room temperature overnight to make dry powder for further experiments.

Electrochemical measurements. Composite electrodes were prepared with $80 \mathrm{wt} \%$ active material, $10 \mathrm{wt} \%$ polyvinylidene fluoride and $10 \mathrm{wt} \%$ acetylene carbon black in NMP ( $N$-methyl-2-pyrrolidone) and cast onto copper current collectors. 2032-type coin cells were assembled in an argon-filled glove box using the composite electrode as the positive electrode and $\mathrm{Li}$ metal as the negative electrode. A Celgard separator 2400 and $1 \mathrm{M} \mathrm{LiPF}_{6}$ electrolyte solution in 1:1 w/w ethylene carbonate/dimethyl carbonate were used to fabricate coin cells. Battery testing was performed on computer controlled systems (Arbin BT2000 and BioLogic VMP3) at $21^{\circ} \mathrm{C}$.

TEM characterization. The in situ TEM electrochemical cell was incorporated into a Nanofactory TEM-STM specimen holder, in which $\mathrm{Fe}_{3} \mathrm{O}_{4}$ nanoparticles dispersed onto a TEM half-grid with amorphous carbon support are analogous to the $\mathrm{Fe}_{3} \mathrm{O}_{4}$-C composite electrode, $\mathrm{Li}$ metal is coated onto a piezo-driven $\mathrm{W}$ probe as the counter electrode, with a thin layer of $\mathrm{Li}_{2} \mathrm{O}$ formed on $\mathrm{Li}$ metal as the solid electrolyte ${ }^{36-38}$. The $\mathrm{Li}$ and $\mathrm{Fe}_{3} \mathrm{O}_{4}$ were loaded onto the holder in an Ar-filled glove box and then transferred to TEM column using a sealed Ar bag to avoid air exposure. During the in situ TEM tests, a constant negative DC potential was applied to $\mathrm{Fe}_{3} \mathrm{O}_{4}$ electrode against the $\mathrm{Li}$ source during the lithiation process, and the lithiation processes were captured by real-time imaging in either TEM or STEM mode. We estimated the average discharge current density across the entire particle surface to be $0.017 \mathrm{~mA} \mathrm{~cm}^{-2}$. The ex situ samples after discharge in coin cells were examined accordingly. The in situ BF-STEM measurements were performed on a JEOL $2100 \mathrm{~F}$ TEM operated at $200 \mathrm{kV}$. We have used a convergence angle of $12 \mathrm{mrad}$ and a collection angle cutoff at $\sim 20 \mathrm{mrad}$. The high-resolution STEM imaging and analytical EELS were conducted on a Hitachi HD2700C STEM operated at $200 \mathrm{kV}$ and equipped with a probe aberration corrector (spatial resolution $<1 \AA$, energy resolution $0.35 \mathrm{eV}$ )

Theoretical calculations. The DFT calculations were performed using the Vienna $A b$ initio Simulation Package (VASP) within the projector augmented-wave approach with the Perdew - Burke - Ernzerhof generalized-gradient approximation. The DFT parameters were consistent with the parameters used in Materials Project $(\mathrm{MP})^{48}$. A Hubbard $U$ term of $5.3 \mathrm{eV}$ is adopted for $\mathrm{Fe}^{2+}$ and $\mathrm{Fe}^{3+}$ in accordance with the MP and the previous testing ${ }^{51,52}$. The ground-state crystal structures of $\mathrm{LiFe}_{3} \mathrm{O}_{4}$ were obtained by enumerating 380 symmetrically distinctive configurations of $\mathrm{Li} / \mathrm{Fe}$ disordering at the $16 \mathrm{c}$ sites using pymatgen ${ }^{49}$. Several magnetic orderings in ferromagnetic, anti-ferromagnetic and ferrimagnetic structures are tested for iron oxide compounds, such as $\mathrm{Fe}_{3} \mathrm{O}_{4}, \mathrm{FeO}, \mathrm{LiFe}_{3} \mathrm{O}_{4}$ and $\mathrm{LiFeO}_{2}$, to identify the lowest energy state (more details provided in Supplementary Fig. 8 and Supplementary Table 1). The voltage plateaus are obtained using the DFT energies of all relevant compounds in the $\mathrm{Li}-\mathrm{Fe}-\mathrm{O}$ ternary space from the $\mathrm{MP}^{50}$.

The phase-field simulation was performed using the electrochemistry theory based on non-equilibrium thermodynamics developed by Bazant et al. ${ }^{53-55}$. The standard phenomenological model based on the Butler-Volmer equation ${ }^{55,56}$ and the Cahn-Hilliard equation ${ }^{23,57}$ was used for modelling the electrochemical kinetics. A homogeneous free-energy was comprised using piecewise functions of polynomial with continuity and differentiability at the segment points. The overpotential and strain energy are also considered in the simulation. More details are shown in Supplementary Figs 9-11, Supplementary Table 2 and Supplementary Methods.

\section{References}

1. Poizot, P., Laruelle, S., Grugeon, S., Dupont, L. \& Tarascon, J. M. Nano-sized transition-metal oxides as negative-electrode materials for lithium-ion batteries. Nature 407, 496-499 (2000).

2. Tarascon, J. M. \& Armand, M. Issues and challenges facing rechargeable lithium batteries. Nature 414, 359-367 (2001).

3. Whittingham, M. S. Lithium Batteries and Cathode Materials. Chem. Rev. 104, 4271-4301 (2004).

4. Goodenough, J. B. \& Kim, Y. Challenges for rechargeable Li batteries. Chem. Mater. 22, 587-603 (2010).

5. Ferg, E., Gummow, R. J., de Kock, A. \& Thackeray, M. M. Spinel anodes for lithium-ion batteries. J. Electrochem. Soc. 141, L147-L150 (1994).

6. Cabana, J., Monconduit, L., Larcher, D. \& Palacin, M. R. Beyond intercalationbased Li-ion batteries: the state of the art and challenges of electrode materials reacting through conversion reactions. Adv. Mater. 22, E170-E192 (2010).

7. Malini, R., Uma, U., Sheela, T., Ganesan, M. \& Renganathan, N. G. Conversion reactions: a new pathway to realise energy in lithium-ion battery-review. Ionics 15, 301-307 (2009).

8. Taberna, P. L., Mitra, S., Poizot, P., Simon, P. \& Tarascon, J. M. High rate capabilities $\mathrm{Fe}_{3} \mathrm{O}_{4}$-based $\mathrm{Cu}$ nano-architectured electrodes for lithium-ion battery applications. Nat. Mater. 5, 567-573 (2006).

9. Mitra, S., Poizot, P., Finke, A. \& Tarascon, J. M. Growth and electrochemical characterization versus lithium of $\mathrm{Fe}_{3} \mathrm{O}_{4}$ electrodes made via electrodeposition. Adv. Funct. Mater. 16, 2281-2287 (2006).

10. Zhou, G. et al. Graphene-wrapped $\mathrm{Fe}_{3} \mathrm{O}_{4}$ anode material with improved reversible capacity and cyclic stability for lithium ion batteries. Chem. Mater. 22, 5306-5313 (2010).

11. Ji, L. et al. $\mathrm{Fe}_{3} \mathrm{O}_{4}$ nanoparticle-integrated graphene sheets for high-performance half and full lithium ion cells. Phys. Chem. Chem. Phys. 13, 7170-7177 (2011)

12. Zhang, W. M., We, X. L., Hu, J. S., Guo, Y. G. \& Wan, L. J. Carbon coated $\mathrm{Fe}_{3} \mathrm{O}_{4}$ nanospindles as a superior anode material for lithium-ion batteries. $A d v$. Funct. Mater. 18, 3941-3946 (2008).

13. He, Y., Huang, L., Cai, J. S., Zheng, X. M. \& Sun, S. G. Structure and electrochemical performance of nanostructured $\mathrm{Fe}_{3} \mathrm{O}_{4}$ /carbon nanotube composites as anodes for lithium ion batteries. Electrochim. Acta 55, 1140-1144 (2010).

14. Xiong, Q. Q. et al. Synthesis of hierarchical hollow-structured single-crystalline magnetite $\left(\mathrm{Fe}_{3} \mathrm{O}_{4}\right)$ microspheres: the highly powerful storage versus lithium as an anode for lithium ion batteries. J. Phys. Chem. C 116, 6495-6502 (2012).

15. Hariharan, S., Saravanan, K., Ramar, V. \& Balaya, P. A rationally designed dual role anode material for lithium-ion and sodium-ion batteries: case study of ecofriendly $\mathrm{Fe}_{3} \mathrm{O}_{4}$. Phys. Chem. Chem. Phys. 15, 2945-2953 (2013).

16. Thackeray, M. M., David, W. I. F. \& Goodenough, J. B. structural characterization of the lithiated iron oxides $\mathrm{Li}_{\mathrm{x}} \mathrm{Fe}_{3} \mathrm{O}_{4}$ and $\mathrm{Li}_{\mathrm{x}} \mathrm{Fe}_{2} \mathrm{O}_{3}(0<\mathrm{x}<2)$. Mater. Res. Bull. 17, 785-793 (1982).

17. Thackeray, M. M. et al. Spinel electrodes for lithium batteries-a review. J. Power Sources 21, 1-8 (1987).

18. Thackeray, M. M. Spinel electrodes for lithium batteries. J. Am. Ceram. Soc. 82, 3347-3354 (1999).

19. Fontcuberta, J., Rodriguez, J., Pernet, M., Longworth, G. \& Goodenough, J. B. Structural and magnetic characterization of the lithiated iron oxide $\mathrm{Li}_{\mathrm{x}} \mathrm{Fe}_{3} \mathrm{O}_{4}$. J. Appl. Phys. 59, 1918-1926 (1986).

20. Sivakumar, V., Kumar, S., Ross, C. A. \& Shao-Horn, Y. Electrochemical lithium insertion of into iron oxide spinel thin films and nanoparticles: changes in structure and magnetic properties. ECS Trans. 2, 1-11 (2007).

21. Islam, M. S. \& Catlow, C. R. A. Lithium insertion into $\mathrm{Fe}_{3} \mathrm{O}_{4}$. J. Solid State Chem. 77, 180-189 (1988)

22. Liu, H. et al. Capturing metastable structures during high-rate cycling of $\mathrm{LiFePO}_{4}$ nanoparticle electrodes. Science 344, 1252817 (2014).

23. Li, Y. et al. Current-induced transition from particle-by-particle to concurrent intercalation in phase-separating battery electrodes. Nat. Mater. 13, 1149-1156 (2014).

24. Arico, A. S., Bruce, P., Scrosati, B., Tarascon, J. M. \& van Schalkwijk, W. Nanostructured materials for advanced energy conversion and storage devices. Nat. Mater. 4, 366-377 (2005).

25. Jamnik, J. \& Maier, J. Nanocrystallinity effects in lithium battery materials: Aspects of nano-ionics Part IV. Phys. Chem. Chem. Phys. 5, 5215-5220 (2003)

26. Malik, R., Burch, D., Bazant, M. \& Ceder, G. Particle size dependence of the ionic diffusivity. Nano Lett. 10, 4123-4127 (2010).

27. Malik, R., Zhou, F. \& Ceder, G. Kinetics of non-equilibrium lithium incorporation in $\mathrm{LiFePO}_{4}$. Nat. Mater. 10, 587-590 (2011).

28. Huang, J. Y. et al. In situ observation of the electrochemical lithiation of a single $\mathrm{SnO}_{2}$ nanowire electrode. Science 330, 1515-1520 (2010).

29. Liu, X. H. et al. In situ atomic-scale imaging of electrochemical lithiation in silicon. Nat. Nanotechnol. 7, 749-756 (2012). 
30. Liu, Y. et al. In situ transmission electron microscopy observation of pulverization of aluminum nanowires and evolution of the thin surface $\mathrm{Al}_{2} \mathrm{O}_{3}$ layers during lithiation-delithiation cycles. Nano Lett. 11, 4188-4194 (2011).

31. McDowell, M. T. et al. Studying the kinetics of crystalline silicon nanoparticle lithiation with in situ transmission electron microscopy. Adv. Mater. 24, 6034-6041 (2012).

32. McDowell, M. T. et al. In situ observation of divergent phase transformations in individual sulfide nanocrystals". Nano Lett. 15, 1264-1271 (2015).

33. Gu, M. et al. In situ tem study of lithiation behaviour of silicon nanoparticles attached to and embedded in a carbon matrix. ACS Nano 6, 8439-8447 (2012).

34. Gu, M. et al. Probing the failure mechanism of $\mathrm{SnO}_{2}$ nanowires for sodium-ion batteries. Nano Lett. 13, 5203-5211 (2013).

35. Nie, A. et al. Atomic-scale observation of lithiation reaction front in nanoscale $\mathrm{SnO}_{2}$ materials. ACS Nano 7, 6203-6211 (2013).

36. $\mathrm{He}, \mathrm{K}$. et al. Sodiation via heterogeneous disproportionation in $\mathrm{FeF}_{2}$ electrodes for sodium-ion batteries. ACS Nano 8, 7251-7259 (2014).

37. He, K. et al. Transitions from near-surface to interior redox upon lithiation in conversion electrode materials. Nano Lett. 15, 1437-1444 (2015).

38. He, K. et al. Sodiation kinetics of metal oxide conversion electrodes: a comparative study with lithiation. Nano Lett. 15, 5755-5763 (2015).

39. Zhu, Y. et al. In situ atomic-scale imaging of phase boundary migration in $\mathrm{FePO}_{4}$ microparticles during electrochemical lithiation. Adv. Mater. 25, 5461-5466 (2013)

40. Niu, J. et al. In situ observation of random solid solution zone in $\mathrm{LiFePO}_{4}$ electrode. Nano Lett. 14, 4005-4010 (2014).

41. Sun, Y. et al. Direct atomic-scale confirmation of three-phase storage mechanism in $\mathrm{Li}_{4} \mathrm{Ti}_{5} \mathrm{O}_{12}$ anodes for room-temperature sodium-ion batteries. Nat. Commun. 4, 1870 (2013).

42. $\mathrm{Lu}, \mathrm{X}$. et al. Lithium storage in $\mathrm{Li}_{4} \mathrm{Ti}_{5} \mathrm{O}_{12}$ spinel: the full static picture from electron microscopy. Adv. Mater. 24, 3233-3238 (2012).

43. Laruelle, S. et al. On the origin of the extra electrochemical capacity displayed by MO/Li cells at low potential. J. Electrochem. Soc. 149, A627-A634 (2002).

44. Ponrouch, A., Taberna, P. L., Simon, P. \& Palacin, M. R. On the origin of the extra capacity at low potential in materials for Li batteries reacting through conversion reaction. Electrochim. Acta 61, 13-18 (2012).

45. Hu, Y. Y. et al. Origin of additional capacities in metal oxide lithium-ion battery electrodes. Nat. Mater. 12, 1130-1136 (2013).

46. Weppner, W. \& Huggins, R. A. Determination of the kinetic parameters of mixed-conducting electrodes and application to the system $\mathrm{Li}_{3} \mathrm{Sb}$. J. Electrochem. Soc. 124, 1569-1578 (1977).

47. Pennycook, S. J. \& Nellist, P. D. Scanning Transmission Electron Microscopy Imaging and Analysis 97-101 (Springer Science \& Business Media, 2011).

48. Jain, A. et al. A high-throughput infrastructure for density functional theory calculations. Comp. Mater. Sci. 50, 2295-2310 (2011).

49. Ong, S. P. et al. Python materials genomics (pymatgen): a robust, open-source python library for materials analysis. Comp. Mater. Sci. 68, 314-319 (2013).

50. Jain, A. et al. The materials project: a materials genome approach to accelerating materials innovation. APL Mater. 1, 011002 (2013).

51. Jain, A. et al. Formation enthalpies by mixing GGA and GGA + U calculations. Phys. Rev. B 84, 045115 (2011).

52. Wang, L., Maxisch, T. \& Ceder, G. Oxidation energies of transition metal oxides within the GGA + U framework. Phys. Rev. B 73, 195107 (2006)

53. Bazant, M. Z. Theory of chemical kinetics and charge transfer based on nonequilibrium thermodynamics. Acc. Chem. Res. 46, 1144-1160 (2013)

54. Bai, P., Cogswell, D. A. \& Bazant, M. Z. Suppression of phase separation in $\mathrm{LiFePO}_{4}$ nanoparticles during battery discharge. Nano Lett. 11, 4890-4896 (2011).

55. Biesheuvel, P. M., van Soestbergen, M. \& Bazant, M. Z. Imposed currents in galvanic cells. Electrochim. Acta 54, 4857-4871 (2009).

56. Newman, J. Electrochemical Systems 2nd edn (Prentice Hall, 1991).
57. Cahn, J. W. \& Hilliard, J. E. Free energy of a nonuniform system. I. interfacial free energy. J. Chem. Phys. 28, 258-267 (1958).

58. Kim, D. et al. Synthesis of uniform ferrimagnetic magnetite nanocubes. J. Am. Chem. Soc. 131, 454 (2009).

59. Dong, A. et al. A generalized ligand-exchange strategy enabling sequential surface functionalization of colloidal nanocrystals. J. Am. Chem. Soc. 133, 998-1006 (2011).

\section{Acknowledgements}

This research used resources of the Center for Functional Nanomaterials, which is a US DOE Office of Science Facility, at Brookhaven National Laboratory under Contract No. DE-SC0012704. X.Y., E.H., K.S., H.G. and X.-Q.Y. were supported by the US DOE, the Assistant Secretary for Energy Efficiency and Renewable Energy, Office of Vehicle Technologies under Contract No. DE-SC00112704. We acknowledge the technical support by Dr Wenqian Xu from Beamline 17-BM-B of Advanced Photon Source at Argonne National Laboratory. S.Z., H.Y. and C.B.M. acknowledge the support of MRSEC award No. DMR-1120901. S.Z. also acknowledges the support of the NatureNet Science Fellowship. Q.M. and Yimei Z. are supported by DOE/BES, Division of Materials Science and Engineering, under Contract No. DE-SC0012704. Those in situ and analytical TEM experiments performed by J.L. and E.A.S. were supported as part of the Center for Mesoscale Transport Properties, an Energy Frontier Research Center supported by the US Department of Energy, Office of Science, Basic Energy Sciences, under award \#DE-SC0012673. Yizhou Z. and Y.M. acknowledge the support of the Minta Martin award at University of Maryland, and the computational resources from Extreme Science and Engineering Discovery Environment (XSEDE) supported by the National Science Foundation Grant No. TG-DMR130142 and from the University of Maryland supercomputing resources. We thank Dr Peng Bai for helpful discussions on phase-field simulaitons.

\section{Author contributions}

K.H., S.Z., D.S. and C.B.M. conceived and designed the experiments. K.H., D.S. and J.L. performed in situ and ex situ TEM experiments. S.Z., H.Y. and C.B.M. synthesized the $\mathrm{Fe}_{3} \mathrm{O}_{4}$ samples. J.L., X.Y., E.H., X.-Q.Y., K.S. and H.G. conducted electrochemical tests. Yizhou Z. and Y.M. performed DFT calculation. Q.M. performed phase-field simulation. K.H. prepared the figures and wrote the manuscript with D.S., S.Z., E.A.S., Yimei Z. and C.B.M. D.S. supervised the project. All the authors participated in discussion of the results.

\section{Additional information}

Supplementary Information accompanies this paper at http://www.nature.com/ naturecommunications

Competing financial interests: The authors declare no competing financial interests.

Reprints and permission information is available online at http://npg.nature.com/ reprintsandpermissions/

How to cite this article: $\mathrm{He}, \mathrm{K}$. et al. Visualizing non-equilibrium lithiation of spinel oxide via in situ transmission electron microscopy. Nat. Commun. 7:11441 doi: 10.1038/ncomms11441 (2016).

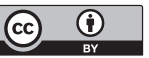

This work is licensed under a Creative Commons Attribution 4.0 International License. The images or other third party material in this article are included in the article's Creative Commons license, unless indicated otherwise in the credit line; if the material is not included under the Creative Commons license, users will need to obtain permission from the license holder to reproduce the material. To view a copy of this license, visit http://creativecommons.org/licenses/by/4.0/ 